\title{
The Examination of Ice Hockey Forward and Backward Skating Mechanics
}

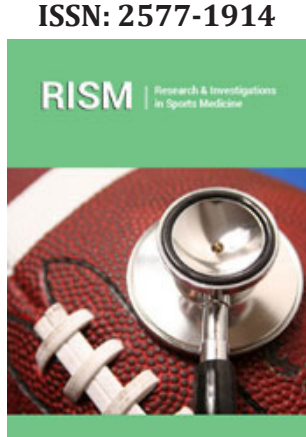

*Corresponding author: Tong-Ching Tom Wu, Department of Movement Arts, Health Promotion and Leisure Studies, Adrian Tinsley Center, Bridgewater State University, USA

Submission: 䟧 March 20, 2020

Published: 阱April 27, 2020

Volume 6 - Issue 3

How to cite this article: Tom $\mathrm{Wu}$, David Pearsall, Pamela J Russell, Yuko Imanaka. The Examination of Ice Hockey Forward and Backward Skating Mechanics. Res Inves Sports Med, 6(3): RISM.000636. 2020. DOI: $10.31031 /$ RISM.2020.06.000636

Copyright@ Tom Wu, This article is distributed under the terms of the Creative Commons Attribution 4.0 International License, which permits unrestricted use and redistribution provided that the original author and source are credited.

\author{
Tom Wu ${ }^{1 *}$, David Pearsall ${ }^{2}$, Pamela J Russell ${ }^{1}$ and Yuko Imanaka ${ }^{1}$ \\ ${ }^{1}$ Department of Movement Arts, Bridgewater State University, USA \\ ${ }^{2}$ Department of Kinesiology and Physical Education, McGill University, Canada
}

\section{Abstract}

Ice hockey is a popular winter sport, and one of the most important skills in ice hockey is skating. Forward and backward skating may appear to be similar, but the body joint movements may be substantially different. The mechanics of backward skating is not well understood in comparison to the forward skating. Therefore, the purpose of this study was to examine the kinematic differences between forward and backward skating. Seven male collegiate ice hockey players participated in the study, and they skated both forward and backward (C-cut) four times each on the ice at their highest intensity from one end of the goal line to the other end of the goal line on the ice. A standard two-dimensional kinematic analysis was conducted to examine the lower body extremity and trunk at the instants of weight acceptance and propulsion. No significant differences in the joint angles were found at the weight acceptance. At propulsion, significant differences were found in the joint angles of hip, knee, ankle and trunk between forward and backward skating. Additionally, the hip angular velocity was significantly different in both the weight acceptance and the propulsion phases. Hence, these findings indicate the importance of understanding mechanical difference between forward and backward skating for developing coaching instruction and prescribe strength and conditioning programs. Future studies are warranted to examine the mechanics of forward and backward skating using a 3D analysis and also on different positional players.

Keywords: Backward; Forward; Hockey; Kinematic; Mechanics; Skating

\section{Introduction}

Ice hockey is a popular sport in North America and Europe, and in 2019 there were approximately 567,900 registered ice hockey players in the United States [1]. Hockey is a fast paced sport requiring the ability to perform lower intensity striding and gliding, interspersed with bursts of high intensity skating. Other major skills performed in hockey include body checking, stick handling, passing and shooting. Many professional coaches, general managers, and scouts have long considered skating as the most important skill of all [2]. From a biomechanical perspective, having fundamental skating mechanics can contribute to fast skating and reduce head and lower body joint injury. Both Marino \& Weese [3], and Pearsall, et al. [4] conducted a kinematic analysis of forward ice skating in hockey. These studies described the ice skating stride as consisting of three functional phases: 1) glide during single support, 2) propulsion during single support, and 3) propulsion during double support. The glide during single support phase coincides with the full extension of the propulsion leg, and the beginning of recovery of the leg back to the support position. The propulsion during the single support phase consists of drawing back the recovery leg while the leg that has been gliding and supporting the body externally rotates at the ankle and begins to push out and back. Finally, the propulsion during double support phase occurs once the recovery leg again contacts the ice under the body, and the propulsion continues with the other leg. This pattern is repeated as forward motion continues. The percentage of time in each stage differs across individuals and will also vary with speed and direction changes. Forward acceleration will continue as long as propulsion continues. Therefore, maintenance of speed is highly related to maximizing the amount of propulsion and minimizing the amount of glide.

The kinematics of forward ice skating have been well examined in the previous literature [4-7]. However, the kinematics of backward ice skating is not well understood because thus far there has been only one research study that has evaluated the mechanics of backward 
ice skating [8]. Mario \& Grasse [8] studied the selected movement characteristics and backward skating velocity. Ten skilled hockey players participated in the study, and the authors found that the backward skating cycle time was 0.86 seconds with a cycle length of $5.65 \mathrm{~m}$. The authors also indicated that the backward skating velocity was $6.57 \mathrm{~m} / \mathrm{s}$, which is approximately $81 \%$ of the forward skating velocity at $8.03 \mathrm{~m} / \mathrm{s}$. Since in this study the camera was suspended from the overhead, the body mechanics of backward skating were not examined. Understanding the body mechanics of backward skating is crucial because it enables players to have proper balance on the ice while skating backward efficiently without falling, which potentially may cause serious head (i.e. concussion) and body joint injuries. Backward skating skill is also particularly important for defensive players in ice hockey. Twist \& Rhodes [9] reported that defensive players play close to $50 \%$ of a game whereas forward players play approximately $35 \%$ of ice time. Hence, a defensive player spends a substantial amount of time on the ice skating backwards during a game. The mechanics of forward and backward skating can be similar but yet substantially different due to the direction of the skating motion. The researcher hypothesized that the players may have a greater lower extremity joint angles in forward skating than backward skating, so the skaters could propel themselves to skate faster with a higher velocity in forward skating than in backward skating. However, due to the lack of empirical evidence on backward skating mechanics, this observation needed to be examined and validated. Therefore, the purpose of this study was to examine and compare both kinematics of forward and backward ice skating in the lower extremity. The results would enable coaches to provide a comprehensive skating instruction for developing minor hockey players and beginners, and also trainers can prescribe a better strength and conditioning program to reduce body joint injury.

\section{Methods}

\section{Participants and Experimental Design}

Seven division II male intercollegiate club hockey players (mean age: $20.6 \pm 2.6$ years old; height: $1.8 \pm 6.6 \mathrm{~m}$; mass: $82.9 \pm 15.7 \mathrm{~kg}$ ) who were free of injury were recruited to participate in the study, and the Institutional Research Board (IRB) approved the study. Written informed consent was obtained from the participants prior to the study. Data collection took place at a local ice arena. Participants were asked to dress in dark, tight-fitting clothing, and to perform their usual off-ice warm up prior to arriving for testing. Upon arrival, the participants were fitted with reflective joint markers on both sides of the body at the acromion of the shoulder, greater trochanter of the hip, the lateral epicondyle of the distal femur of the knee, the lateral malleolus at the ankle, the heel, and the 5th metatarsal-phalangeal joint. Participants wore hockey skates and gloves and carried a stick. Participants were instructed to skate both forward and backward four times each at their highest intensity from one end of the goal line to the other end of the goal line on the ice $(54.25 \mathrm{~m})$. Participants had three minutes rest between each trial and five minutes between each type of skate. The order of the skate (forward or backward) was randomized to reduce any order effect. A total of 56 trials ( 7 participants $x 2$ types of skate $\mathrm{x} 4$ trials/ skate) were collected in the study. Three best trials with similar lower body kinematic joint angles (hip, knee and ankle) from each participant were selected, so a total of 42 trials were used for the statistical analysis. Data collection lasted approximately one hour in duration for each participant. Video of the skating trials were collected using standard two-dimensional videography with a high-speed JVC (Model: GR-D371U) digital video camera operating at $60 \mathrm{~Hz}$. The camera was positioned at the centerred line to capture the sagittal view of the skating motion with two calibration poles set up on the ice to create a field of view of $7.90 \mathrm{~m}$ horizontally. Additionally, a 650W artificial spot light was used in conjunction with the camera to assist joint marker identification. Video trials were analyzed using APASTM (Ariel Performance Analysis System). Previous literature examined instants of weight acceptance (foot fully contact with the ice in the beginning of stance phase) and propulsion (toe off the ice in the beginning of swing phase) to understand the mechanics of forward skating [7]. This research study used the same instants for forward and backward skating analysis. Since backward skating skill (C-cut) does not have a swing phase only a stance phase due to the foot contact with the ice the whole time, the instants of weight acceptance and propulsion could not visually be identified from video trials. Hence, this study used the instants of maximum hip flexion as the weight acceptance and maximum hip extension as the propulsion for analysis because these two instants best represented the motion occurring at the weight acceptance and at propulsion, and it also provided objectivity and consistency across trials for data analysis. The coordinate data were then smoothed with a digital filter function with appropriate cut off frequencies $(\mathrm{x}=8 \mathrm{~Hz}$ and $\mathrm{y}=8 \mathrm{~Hz})$. Smoothed data were used in the kinematic analysis to generate joint angles, velocities and accelerations at the hip, knee, and ankle intersegment joints and as well as the trunk segment (with respect to the horizontal axis). A paired samples t-test with $\alpha=0.05$ was conducted using SPSS (v. 28) software to examine significant differences between forward and backward skating for the body joint kinematic variables.

\section{Results}

Kinematics of lower body joint angles, velocities and accelerations were compared between forward and backward skating skills. At the instant of weight acceptance there were no significant differences in the trunk and lower body joint angles and velocities (Tables 1-3), but significant differences were observed in the hip and knee joint angular velocities (Table 2). At the instant of propulsion significant differences were observed in the trunk and all lower body joint angles (Table 4). Additionally, significant differences were observed in the angular velocities of the hip, ankle and trunk (Table 5). A significant difference was observed in the angular acceleration of the knee joint (Table 6). 
Table 1: Angular displacement between forward and backward skating at weight acceptance.

\begin{tabular}{|c|c|c|}
\hline \multirow{2}{*}{ Joint } & Forward vs Backward & \multirow{2}{*}{$\mathbf{p}$} \\
\cline { 2 - 2 } & Angular Displacement Mean (SD) & \\
\hline Hip & $85.4(10.7)$ vs $83.8(9.4)$ & 0.683 \\
\hline Knee & $96.4(9.0)$ vs $93.4(8.3)$ & 0.327 \\
\hline Ankle & $78.9(7.7)$ vs $78.7(10.0)$ & 0.949 \\
\hline Trunk & $50.6(8.3)$ vs $51.7(9.4)$ & 0.625 \\
\hline
\end{tabular}

Statistical significant at $\mathrm{p}<0.05$

Table 2: Angular velocity between forward and backward skating at weight acceptance.

\begin{tabular}{|c|c|c|}
\hline \multirow{2}{*}{ Joint } & Forward vs Backward & \multirow{2}{*}{ p } \\
\cline { 2 - 2 } & Angular Velocity Mean (SD) $/ \mathbf{s}$ & \\
\hline Hip & $68.3(37.0)$ vs $-38.3(62.5)$ & $.017^{*}$ \\
\hline Knee & $36.2(68.2)$ vs $-88.0(59.4)$ & $.020^{*}$ \\
\hline Ankle & $-14.1(69.2)$ vs $-21.2(82.4)$ & 0.856 \\
\hline Trunk & $-4.3(33.6)$ vs $24.5(35.5)$ & 0.275 \\
\hline
\end{tabular}

*Statistical significant at $\mathrm{p}<0.05$

Table 3: Angular acceleration between forward and backward skating at weight acceptance.

\begin{tabular}{|c|c|c|}
\hline Joint & $\begin{array}{c}\text { Forward vs Backward } \\
\text { Angular Acceleration Mean (SD) }{ }^{\circ} \mathbf{s}^{2}\end{array}$ & $\boldsymbol{p}$ \\
\hline Hip & $3677.6(2074.9)$ vs $3647.3(2293.7)$ & 0.842 \\
\hline Knee & $-45.3(2493.6)$ vs $2621.7(1867.3)$ & .053 \\
\hline Ankle & $-1349.7(7687.9)$ vs $-195.5(1819.5)$ & .736 \\
\hline Trunk & $2602.0(2418.9)$ vs $861.8(776.5)$ & 0.104 \\
\hline
\end{tabular}

*Statistical significant at $\mathrm{p}<0.05$

Table 4: Angular displacement between forward and backward skating at propulsion.

\begin{tabular}{|c|c|c|}
\hline \multirow{2}{*}{ Joint } & Forward vs Backward & \multirow{2}{*}{$\boldsymbol{p}$} \\
\cline { 2 - 3 } & Angular Displacement Mean (SD) & \\
\hline Hip & $128.2(11.1)$ vs $113.3(10.8)$ & $.006^{*}$ \\
\hline Knee & $137.2(12.3)$ vs $123.8(3.0)$ & $.025^{*}$ \\
\hline Ankle & $90.2(10.7)$ vs $75.4(11.6)$ & $.039^{*}$ \\
\hline Trunk & $27.2(7.5)$ vs $49.6(8.8)$ & $.000^{*}$ \\
\hline
\end{tabular}

*Statistical significant at $\mathrm{p}<0.05$

Table 5: Angular velocity between forward and backward skating at propulsion.

\begin{tabular}{|c|c|c|}
\hline \multirow{2}{*}{ Joint } & Forward vs Backward & \multirow{2}{*}{$\boldsymbol{p}$} \\
\cline { 2 - 2 } & Angular Velocity Mean (SD) $/ \mathbf{s}$ & \\
\hline Hip & $275.5(177.2)$ vs $6.8(24.0)$ & $.008^{*}$ \\
\hline Knee & $-68.1(223.8)$ vs $36.8(59.2)$ & .209 \\
\hline Ankle & $533.7(264.8)$ vs $44.7(67.0)$ & $.008^{*}$ \\
\hline Trunk & $68.7(18.5)$ vs $-35.3(49.7)$ & $.001^{*}$ \\
\hline
\end{tabular}

*Statistical significant at $\mathrm{p}<0.05$
Table 6: Angular acceleration between forward and backward skating at propulsion.

\begin{tabular}{|c|c|c|}
\hline \multirow{2}{*}{ Joint } & Forward vs Backward & \multirow{2}{*}{$\boldsymbol{p}$} \\
\cline { 2 - 3 } & Angular Velocity Mean (SD) ${ }^{\circ} / \mathbf{s}$ & .534 \\
\hline Hip & $-7225.0(6006.4)$ vs $-5459.4(3156.0)$ & $.049^{*}$ \\
\hline Knee & $-13153.9(6138.3)$ vs $-6488.0(3214.2)$ & .145 \\
\hline Ankle & $-5494.4(9743.9)$ vs $617.2(2351.5)$ & 0.18 \\
\hline Trunk & $904.4(1419.3)$ vs $-427.8(1511.7)$ & \\
\hline
\end{tabular}

*Statistical significant at $\mathrm{p}<0.05$

\section{Discussion and Implications}

The motion between forward and backward skating may be similar but yet substantially different. The skill of forward skating is characterized by both swing and support phases, and each phase is further divided into single and double periods of leg support [4], whereas, the skill of backward skating (C-cut technique) consists of continuously double leg contact with the ice surface during the support phase only and alternately shifting leg from one side to the other [8]. In this study similar lower body jointangles and trunk angle were found at the instant of weight acceptance between forward and back skating. However, forward skating skill showed greater hip and knee angular velocities comparing to backward skating skill. The difference in the hip and knee joint angular velocities may explain why forward skating $(10.1 \pm 1.9 \mathrm{~m} / \mathrm{s})$ has a higher skating velocity than backward skating $(8.2 \pm 0.2 \mathrm{~m} / \mathrm{s})$. In addition, at the instant of propulsion skaters showed significantly greater hip, knee and ankle extension movements in forward skating than backward skating. Also, the trunk angle in forward skating is significantly less than backward skating, which indicates the skaters leaned their upper body forward while skating forward. The forward trunk lean allowed the skaters to almost fully extend their push off leg at propulsion to propel their body forward at the end of stance phase. This also explains why at the instant of propulsion a significantly higher amount of angular velocity was observed in the hip, ankle and trunk in forward skating than backward skating, which further contributed to higher forward skating velocity. The knee joint also showed a higher angular velocity but with a negative knee angular velocity, and this indicated that at propulsion the knee began to flex while the hip and ankle continued to extend, perhaps to allow a quicker leg recovery in the skating cycle.

There is limited literature that examines the mechanics of the backward C-cut skating skill, so this study provides an important understanding of lower body mechanics and of trunk position in performing the backward skating skill. During the weight acceptance of the backward skating, the hip, knee, and ankle joints were $83.8 \pm 9.4^{\circ}, 93.4 \pm 8.3^{\circ}, 78.7 \pm 10.0^{\circ}$, respectively, and the trunk segment was $51.7 \pm 9.4^{\circ}$. During the propulsion phase of the backward skating, the hip, knee, and ankle joints were $113.3 \pm$ $10.8^{\circ}, 123.8 \pm 3.0^{\circ}, 75.4 \pm 11.6^{\circ}$, respectively, and the trunk segment was $49.6 \pm 8.8^{\circ}$. The lower body joint angles for the backward C-cut 
skating were significantly lower than the forward skating. This indicates the hip, knee and ankle joints flexed more because in the backward C-cut skating skill the foot does not leave the ice since there is no swing phase. Thus, the leg is not fully extended at the propulsion phase, which minimizes the ability for the foot to push off in order to generate greater power and skating velocity.

Due to the limited previous literature on skating mechanics, the authors have selected literature with similar kinematic analyses (i.e. joint angles were defined the same way.), which enabled the authors to compare the current study with previous research work. In this study the joint kinematics in forward skating at weight acceptance were similar with Hagg, Wu, and Gervais (2007) study of trunk, knee and ankle joint angles $\left(38^{\circ}, 110^{\circ}\right.$, and $67^{\circ}$, respectively) and the McPherson et al. [6] study of hip and knee joint angles $\left(91^{\circ}\right.$ and $103^{\circ}$, respectively). Also, similar results were found in forward skating at propulsion when comparing to McPherson et al. [6] study of hip, knee and ankle joint angles $\left(149^{\circ}, 156^{\circ}\right.$ and $99^{\circ}$, respectively). There were some limitations that should be considered. This research study was conducted in two dimensions rather than in three dimensions. Since the main joint movements occurred in the sagittal plane of motion as indicated by Upjohn et al. [7] for forward skating, this study provided important and practical findings on comparisons between forward and backward skating mechanics. Moreover, this study evaluated seven collegiate hockey players, and there were five forward and two defensive players. Defensive players may have stronger backward skating skills than forward players. It would be interesting to examine if both forward and defensive players demonstrate same or different skating mechanics. Future studies are warranted to conduct a three-dimensional analysis to compare both forward and backward skating skills and for different positional players.

\section{Conclusion}

This research study compared the kinematics of forward and backward skating for male varsity ice hockey players. The results showed that the skaters demonstrated significantly greater hip, knee, and ankle extension movements at propulsion in forward skating than backward skating. Additionally, the skaters showed greater forward trunk lean in forward skating than in backward skating which may maximize their skating velocity. These findings indicate the importance of understanding the proper mechanics of skating for both forward and backward skating skills for developing coaching instruction. Further, these enable practitioners to prescribe strength and conditioning programs that match lower body joint action for both forward and backward skating skills. Future studies are warranted to conduct a three-dimensional analysis in both forward and backward skating with different positional players.

\section{Acknowledgement}

The authors would like to thank Bridgewater State University (BSU) men's ice hockey players for participating in the study. In addition, the authors acknowledge the research development funding support from the BSU Canadian Studies Council and the BSU Center for the Advancement of Research and Scholarship.

\section{References}

1. USA Hockey (2019) Membership Statistics.

2. Hansen H, Reed A (1979) Functions and On-ice Competencies of a High Caliber Hockey Player--a Job Analysis. In: J Terauds \& HJ Gros (Eds.), Science in Skiing, Skating, and Hockey. Proceedings of the International Symposium of Biomechanics in Sports. Del Mar, Academic Publishers, California, USA, pp. 107-115.

3. Marino GW, Weese RG (1979) A kinematic analysis of the ice skating stride. In: J Terauds \& HJ Gros (Eds.), Science in Skiing, Skating, and Hockey. Proceedings of the International Symposium of Biomechanics in Sports (pp. 65- 74). Del Mar, Academic Publishers, California, USA, pp. 65-74.

4. Pearsall DJ, Turcotte RA, Murphy SD (2000) Biomechanics of ice hockey. In: WE Garrett \& DT Kirkendall (Eds.), Exercise and Sport Science. Lippencott, Williams \& Wilkins, Philadelphia, USA, pp. 675-692.

5. Marino G W (1983) Selected mechanical factors associated with acceleration in ice skating. Research Quarterly for Exercise and Sport 54(3): 234-238.

6. McPherson MN, Wrigley A, Montelpare WJ (2004) The biomechanical characteristics of development-age hockey players: Determining the effects of body size on the assessment of skating technique. Safety in Ice Hockey pp. 272-287.

7. Upjohn T, Turcotte R, Pearsall DJ, Loh J (2008) Three-dimensional kinematics of the lower limbs during forward ice skating. Sports Biomech 7(2): 206-221.

8. Marino GW, Grasse J (1993). Analysis of selected mechanics of the backward c-cut ice skating stride. Proceedings of XI International Symposium on Biomechanics in Sports (pp. 159-162). Amherst, MA: International Society of Biomechanics in Sports.

9. Twist P, Rhodes T (1993) A physiological analysis of ice hockey positions. National Strength and Conditioning Association Journal 15(6): 44-46. 